\title{
Shaping ability of NiTi rotary versus stainless steel hand instruments in simulated curved canals
}

\author{
Shiva Sadeghi
}

DMD, MSD, Department of Endodontics, Dental School/Dental Research Center, Guilan University of Medical Sciences, Rasht, Iran

Correspondence:

Endodontic department

Dental School/dental research center, Guilan University of Medical Sciences, Opp. Pardis Hotel, Rasht, Iran dental@gums.ac.ir

Received: 06/03/2010 Accepted: 04/06/2010

Sadeghi S. Shaping ability of NiTi rotary versus stainless steel hand instruments in simulated curved canals Med Oral Patol Oral Cir Bucal. 2011 May 1;16 (3):e454-8.

http://www.medicinaoral.com/medoralfree01/v16i3/medoralv16i3p454.pdf

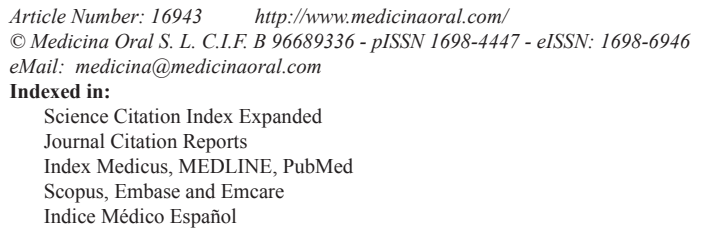

\begin{abstract}
Objectives: The purpose of this in-vitro study was to compare shaping ability of NiTi rotary Mtwo and FlexMaster with stainless steel hand K-Flexofile in simulated curved root canals.

Study Design: Forty-five simulated canals in resin blocks were prepared with Mtwo, FlexMaster and stainless steel hand K-Flexofile (15 blocks in each group). Using pre- and post- instrumentation images, straightening of the canal curvature was determined with a computer image analysis program. Material removal was measured at 5 measuring points, beginning $1 \mathrm{~mm}$ from the apex. Changes of working length (WL) were also recorded. The data were analyzed statistically using paired T-test and one way ANOVA.

Results: The mean material removal from the inner canal wall was different from the outer canal wall at all measuring points for each system $(\mathrm{p}<0.0001)$ except for FlexMaster at WL-9 $(\mathrm{p}=0.123)$ and K-Flexofile at WL-5 ( $\mathrm{p}$ $=0.093$ ). The mean ratio of material removal (inner/outer) at all measuring points was different for all systems except for FlexMaster and Mtwo at 3, $5 \mathrm{~mm}(\mathrm{p}=0.984, \mathrm{p}=0.242)$, and K-Flexofile and rotary systems at 1, $3 \mathrm{~mm}$ from the apex $(p=0.565, p=0.218)(p=0.794, p=0.693)$. A mean loss of working length of $0.02 \mathrm{~mm}$ for Mtwo and $0.01 \mathrm{~mm}$ for FlexMaster and K-Flexofile was measured although the difference was not significant.

Conclusions: In the apical points of the curvature $(1,3 \mathrm{~mm})$, there was no significant difference between three systems. At point $5 \mathrm{~mm}$ from the apex, K-Flexofile remained better centered, while in the coronal points (7, $9 \mathrm{~mm})$ NiTi rotary systems achieved better canal geometry.
\end{abstract}

Key words: Root canal preparation, rotary instruments, hand instruments. 


\section{Introduction}

One of the most important principles of shaping the root canal system is to maintain the original canal anatomy during a continuously tapering preparation. Over the past decade, many instruments, devices and instrumentation techniques have been introduced to achieve this goal. A number of studies have demonstrated that the NiTi rotary instruments allow more rapid, more conservative, rounder canal shaping and better cleaning than hand instruments (1-4). Thus, rotary preparation of the canal system has gained more attention than traditional hand instrumentation as for the cleaning and shaping of the root canal system. The purpose of this study was to compare the shaping ability of two new NiTi rotary systems with a stainless steel hand file in simulated curved root canals.

The new Mtwo instruments (VDW, Munich, Germany) should be used in a single length technique. That means that all files of the instrumentation sequence should be used to the full length of the root canal. Mtwo instruments have an S-shaped cross-sectional design and a non-cutting safety tip. These instruments have a positive rake angle with two cutting edges, which are claimed to cut dentine effectively. Moreover, Mtwo instruments have an increasing pitch length from the tip to the shaft. This design is alleged to have two functions: (a) to eliminate threading and binding in continuous rotation and (b) to reduce the transportation of debris towards the apex. The basic series of Mtwo instruments comprises eight instruments with tapers ranging between $4 \%$ and $7 \%$ and sizes from 10 to 40 (5).

Shaping ability of rotary Mtwo system has been compared with several NiTi rotary instruments $(5,6)$. However, there is no published research to compare shaping ability of NiTi rotary Mtwo with stainless steel hand KFlexofile and NiTi rotary Flex Master.

The Flex Master instruments (VDW, Munich, Germany) are made of 55-nitinol, with cutting edges machined into a round blank, resulting in a convex cross-section that is characterized by three equally spaced cutting edge, which are very similar to k-type blades. The instrument does not have radial lands or U-shaped blades. The Flex Master instruments have flattened non-cutting tips and a rounded transitional angle (3). The manufacturer recommends enlarging that root canals with these instruments using the crown-down technique.

K-Flexofiles were selected as stainless steel hand instruments, because these files also have a non-cutting tip without transition angle and a number of NiTi rotary systems were compared with these files in previous studies $(3,4)$. Thus, the primary aim of this in-vitro study was to compare shaping ability of NiTi rotary Mtwo and Flex Master with stainless steel hand K-Flexofile in simulated curved root canals. The second aim was to compare shaping ability of NiTi rotary Mtwo with FlexMaster system.

\section{Material and Methods}

Simulated curved canals made of clear polyester resin (VDW, Munich, Germany) with $35^{\circ}$ Schneider angle were used to assess instrumentation according to previously described method $(3,5)$. The radius of curvature was calculated about $7.8 \mathrm{~mm}$. The diameter and the taper of all simulated canals were equivalent to an ISO standard size 15 root canal instrument. Canals were $19 \mathrm{~mm}$ long, the straight part being $5 \mathrm{~mm}$ and the curved part $7 \mathrm{~mm}$.

Prior to instrumentation, one of the root canal walls of the simulated canals were colored by inserting blue pelikan ink (Pelikan, Hanover, Germany). Pre-instrumentation canal shape was taken in a standardized manner using a Nikon 801s camera and stored in a computer.

The specimens were divided into three experimental groups $(n=15)$. The simulated canals were prepared with either Mtwo, FlexMaster or hand K-Flexofile instruments. Glycerin was used as lubricant, and a total of $10 \mathrm{ml}$ normal saline was used repeatedly after the use of each instrument. Each instrument was used to enlarge five canals only. These five blocks were defined as a set. The order of use of the three instrument types within a set was rotated. Canals were prepared by an operator experienced in preparation with the different types of instrument. Only five resin blocks were instrumented at a time to minimize operator fatigue and bias towards one of the three instrumentation techniques.

Rotary instruments were set into permanent rotation with a 4:1 reduction handpiece (Nakamura Dental MFG, Japan) powered by a torque-limited electric motor (Endo IT motor; VDW, Munich, Germany). For each file the individual torque limit and rotational speed programmed in the file library of the Endo IT motor were used. All canals were enlarged to an apical size of 30 according to the manufacturer's instructions. The following instrumentation sequence was used with different instruments: All Mtwo instruments were used to the full length of the canals using a gentle in-and-out motion. The instrumentation sequence was: 10/0.04 (WL), 15/0.05 (WL), 20/0.06 (WL), 25/0.06 (WL), 30/0.05 (WL). Once the instrument had achieved the end of the canal and had rotated freely, it was removed.

All Flex Master instruments were used in a crown down manner using a gentle in-and-out motion. Instruments were withdrawn when resistance was felt and changed for the next instrument: 20/0.06 (one-half of WL), 30/0.04 (one-half of WL), 25/0.04 (two-third of WL), 20/0.04 (WL), 20/0.02 (WL, 25/0.02 (WL), 30/0.02 (WL). Once the instrument had achieved the end of the canal and had rotated freely, it was removed.

K-Flexofile hand instruments (Dentsply Maillefer, Ballaigues, Switzerland) were used in step back manner: 15/0.02 (WL), 20/0.02 (WL), 25/0.02 (WL), 30/0.02 (WL), 35/0.02 (WL-1), 30/0.02 (WL), 40/0.02 (WL-2) ,30/0.02 (WL), 45/0.02 (WL-3), 30/0.02 (WL). 
All canals were colored after canal preparation and postinstrumentation images were provided and scanned. Superimposition of pre- and post-instrumentation scanned images was aided by three holes placed in the sides of resin blocks. The amount of resin removal or the difference between the canal configuration before and after instrumentation was determined both for the inner and outer side of the curvature in 1,3,5,7,9 $\mathrm{mm}$ from the apical ending of the canal using the Photoshop 5 program. Measurement of the canals was carried out by a second examiner who was unaware of the experimental groups. Changes of working length were determined by subtracting the final length of each canal after preparation from the original length $(19 \mathrm{~mm})$.
The data were analyzed by using one way ANOVA and Tukey HSD test. The level of statistical significance was set at $\mathrm{p}<0.05$.

\section{Results}

During preparation of the canals, only one stainlesssteel hand K-Flexofile fractured. Therefore, the following results are based on the remaining 14 canals in stainless steel hand K-Flexofile group and 15 canals for each rotary group. The mean amount of material removal from the outer and the inner side of the curvature is shown in table 1. Statistical analysis using paired T-test showed that the mean material removal from the inner canal wall was different from the outer canal wall at all

Table 1. Mean and standard deviation of the material removed $(\mathrm{mm})$ from inner and outer canal walls at different measuring points.

\begin{tabular}{|c|c|c|c|c|c|c|}
\hline \multirow{3}{*}{ Instrument } & \multirow{5}{*}{ Wall } & \multicolumn{5}{|c|}{ Distance from the apex (mm) } \\
\cline { 3 - 7 } & Inner & $0.01(0.002)$ & $0.02(0.002)$ & $0.06(0.004)$ & $0.27(0.01)$ & $0.34(0.009)$ \\
\cline { 2 - 8 } & Outer & $0.11(0.002)$ & $0.17(0.01)$ & $0.25(0.01)$ & $0.14(0.003)$ & $0.11(0.007)$ \\
\cline { 2 - 8 } & P-value & $\mathrm{P}<0.0001$ & $\mathrm{P}<0.0001$ & $\mathrm{P}<0.0001$ & $\mathrm{P}<0.0001$ & $\mathrm{P}<0.0001$ \\
\hline \multirow{5}{*}{ Mtwo } & Inner & $0.01(0.002)$ & $0.03(0.003)$ & $0.07(0.004)$ & $0.18(0.002)$ & $0.19(0.11)$ \\
\cline { 2 - 8 } & Outer & $0.16(0.003)$ & $0.24(0.02)$ & $0.32(0.01)$ & $0.23(0.003)$ & $0.14(0.002)$ \\
\cline { 2 - 8 } & P-value & $\mathrm{P}<0.0001$ & $\mathrm{P}<0.0001$ & $\mathrm{P}<0.0001$ & $\mathrm{P}<0.0001$ & $\mathrm{P}=0.123$ \\
\hline \multirow{5}{*}{ K-Flexofile } & Inner & $0.03(0.04)$ & $0.08(0.12)$ & $0.3(0.01)$ & $0.44(0.04)$ & $0.36(0.04)$ \\
\cline { 2 - 8 } & Outer & $0.27(0.025)$ & $0.47(0.03)$ & $0.29(0.009)$ & $0.11(0.16)$ & $0.07(0.009)$ \\
\cline { 2 - 8 } & P-value & $\mathrm{P}<0.0001$ & $\mathrm{P}<0.0001$ & $\mathrm{P}=0.093$ & $\mathrm{P}<0.0001$ & $\mathrm{P}<0.0001$ \\
\hline
\end{tabular}

Table 2. Mean and standard deviation of the inner/outer material removed $(\mathrm{mm})$ at different measuring points.

\begin{tabular}{|c|c|c|c|c|c|}
\hline \multicolumn{7}{|c|}{ Distance from the apex (mm) } \\
\hline Instrument & $\mathbf{1}$ & $\mathbf{3}$ & $\mathbf{5}$ & 7 & $\mathbf{9}$ \\
\hline $\begin{array}{c}\text { M two } \\
\text { Inner/Outer }\end{array}$ & $0.16(0.02)$ & $0.13(0.01)$ & $0.24(0.02)$ & $1.89(8.68)$ & $3.15(0.25)$ \\
\hline $\begin{array}{c}\text { FlexMaster } \\
\text { Inner/Outer }\end{array}$ & $0.07(0.01)$ & $0.14(0.02)$ & $0.22(0.01)$ & $0.78(0.01)$ & $1.33(0.79)$ \\
\hline $\begin{array}{c}\text { K-Flexofile } \\
\text { Inner/Outer }\end{array}$ & $0.11(0.15)$ & $0.18(0.27)$ & $1.02(0.05)$ & $5.77(1.69)$ & $5.04(0.87)$ \\
\hline P-value & $\mathrm{P}=0.03$ & $\mathrm{P}=0.693$ & $\mathrm{P}=0.0001$ & $\mathrm{P}=0.0001$ & $\mathrm{P}=0.0001$ \\
\hline
\end{tabular}


measuring points for each system $(\mathrm{p}<0.0001)$ except for FlexMaster at WL-9 $(\mathrm{p}=0.123)$ and K-Flexofile at WL-5 $(p=0.093)$.

The one way ANOVA test revealed that there was a significant difference between the mean ratios of material removal (inner/outer) among the three systems at all measuring points except for WL-3 mm ( $\mathrm{p}=0.693)$ (Table 2). Tukey HSD test showed that the mean ratio of material removal at all measuring points was different for all systems except for FlexMaster and Mtwo at 3, 5 $\mathrm{mm}(\mathrm{p}=0.984, \mathrm{p}=0.242)$, and K-Flexofile and rotary systems at $1,3 \mathrm{~mm}$ from the apex $(\mathrm{p}=0.565, \mathrm{p}=0.218)$ $(\mathrm{p}=0.794, \mathrm{p}=0.693)$.

A mean loss of working length of $0.02 \mathrm{~mm}$ for Mtwo and $0.01 \mathrm{~mm}$ for FlexMaster and K-Flexofile was measured although the difference was not significant.

\section{Discussion}

In the present study, standard resin blocks were used for evaluating the shaping ability of the instruments. Using standard resin blocks under strictly controlled laboratory conditions allow a direct comparison of the shaping ability of different instruments (7). Canal shape, size, taper and curvature are standardized in resin blocks and their advantages have been extensively discussed (8). However, the use of simulated canals in resin blocks does not reflect the action of the instrument in root canal of real teeth because of the differences in the surface texture, hardness and cross-section (7).

When comparing the shaping abilities of different preparation techniques or different root canal instruments, it is important to have a similar apical preparation diameter (9). Thus, in this study the master apical file or the final apical preparation diameter was considered similar for all experimental groups (a size of 30).

According to the results of the present study, all systems removed more material on the outer side of the curvature in the apical 1-3 $\mathrm{mm}$ from the apex. Previously published reports (10-12) have also confirmed that any instrument in a curved canal always tries to straighten itself. It therefore cuts straight ahead and does most of its work removing material on the outside of the curve in the apical region. The mean material removal from the inner to outer canal walls for K-Flexofile system at point $5 \mathrm{~mm}$ from the apex showed statistically significant difference with rotary systems. In another words, stainless-steel hand K-Flexofile remained better centered at this level than rotary instruments. This may be owing to the root canal preparation with instruments of greater taper, because they are stiffer compared with those of ISO taper $(2,13)$. In the coronal 7 and 9 $\mathrm{mm}$ level, hand Flexofile system removed significantly more material from the inner canal wall than both rotary systems. Schäfer et al. showed that with the exception of three measuring points, significant differences occurred between these two different instruments and canals prepared with FlexMaster instruments remained better centered compared with those enlarged with KFlexofiles (3).

Furthermore, the results of several studies concerning the shaping ability of different rotary nickel-titanium instruments and stainless steel hand files were different. Using either resin blocks or extracted human teeth, several studies reported no significant differences between NiTi rotary and stainless steel files $(14,15)$ while others showed that NiTi rotary instruments provided better canal shaping (1-4). Different results obtained with the same instruments and techniques may be owing to variables such as operators and their experiences, the characteristics of the samples, study design and the limitations of the used techniques for evaluating shaping ability.

In addition, our results showed that FlexMaster instruments created a gradual constant transportation toward the outer aspect of the curvature in all measuring points and remained more centered at $9 \mathrm{~mm}$ from the apex. There was no significant difference between shaping ability of NiTi rotary Mtwo and FlexMaster system except for point WL-1. The NiTi rotary Mtwo system acts better than FlexMaster system at point $1 \mathrm{~mm}$ from the apex. This finding can not be compared with the existing data because so far no published reports on the cleaning efficacy of Mtwo versus FlexMaster instruments are available.

In this study, it was possible to control the working distance well in all experimental groups. This finding has been confirmed with several observations $(2,5,13,16)$.

\section{References}

References with links to Crossref - DOI

1. Glossen CR, Haller RH, Dove SB, del Rio CE. A comparison of root canal preparations using $\mathrm{Ni}-\mathrm{Ti}$ hand, Ni-Ti engine-driven, and K-Flex endodontic instruments. J Endod. 1995;21:146-51.

2. Kum KY, Spängberg L, Cha BY, Il-Young J, Msd, Seung-Jong L, Chan-Young L.

Shaping ability of three ProFile rotary instrumentation techniques in simulated

resin root canals. J Endod. 2000;26:719-23.

3. Schäfer E, Lohmann D. Efficiency of rotary nickel-titanium FlexMaster instruments compared with stainless steel hand K-Flexofile-Part 1. Shaping ability in simulated curved canals. Int Endod J. 2002;35:505-13.

4. Schäfer E. Shaping ability of Hero 642 rotary nickel-titanium instruments and stainless steel hand K-Flexofiles in simulated curved root canals. Oral Surg Oral Med Oral Pathol Oral Radiol Endod. 2001;92:215-20.

5. Schäfer E, Erler M, Dammaschke T. Comparative study on the shaping ability and cleaning efficiency of rotary Mtwo instruments. Part 1. Shaping ability in simulated curved canals. Int Endod J. 2006;39:196-202.

6. Bonaccorso A, Cantatore G, Condorelli GG, Schäfer E, Tripi TR. Shaping ability of four nickel-titanium rotary instruments in simulated S-shaped canals. J Endod. 2009;35:883-6.

7. Peters OA. Current challenges and concepts in the preparation of root canal systems: a review. J Endod. 2004;30:559-67.

8. Schäfer E, Tepel J, Hoppe W. Properties of endodontic hand in- 
struments used in rotary motion. Part 2. Instrumentation of curved canals. J Endod. 1995;21:493-7.

9. Bergmans L, Van Cleynenbreugel J, Beullens M, Wevers M, Van Meerbeek B, Lambrechts P. Progressive versus constant tapered shaft design using NiTi rotary instruments. Int Endod J. 2003;36:288-95. 10. Lentine FN. A study of torsional and angular deflection of endodontic files and reamers. J Endod. 1979;5:181-91.

11. Weine FS, Kelly RF, Lio PJ. The effect of preparation procedures on original canal shape and on apical foramen shape. J Endod. 1975;1:255-62.

12. Lim KC, Webber J. The effect of root canal preparation on the shape of the curved root canal. Int Endod J. 1985;18:233-9.

13. Thompson SA, Dummer PM. Shaping ability of Hero 642 rotary nickel-titanium instruments in simulated root canals: Part 2. Int Endod J. 2000;33:255-61.

14. Kosa DA, Marshall G, Baumgartner JC. An analysis of canal centering using mechanical instrumentation techniques. J Endod. 1999;25:441-5.

15. Esposito PT, Cunningham CJ. A comparison of canal preparation with nickel-titanium and stainless steel instruments. J Endod. 1995;21:173-6.

16. Martín-Micó M, Forner-Navarro L, Almenar-García A. Modification of the working length after rotary instrumentation: a comparative study of four systems. Med Oral Patol Oral Cir Bucal. 2009;14:E153-7. 